\title{
Reparación simbólica para mujeres trans víctimas del conflicto armado: ¿por qué no se ha iniciado?
}

\author{
Laura Catalina Tovar Bohórquez ${ }^{*}$ \\ (1c.tovar114@uniandes.edu.co)
}

Artículo de reflexión recibido el 28/11/2014 y aprobado el 19/12/2014

\begin{abstract}
Cómo citar este artículo:
TOVAR BOHÓRQUEZ, Laura Catalina (2014). "Reparación simbólica para mujeres trans víctimas del conflicto armado: ¿por qué no se ha iniciado?”. En: Transpasando Fronteras, núm. 6, pp. 83-98. Cali, Colombia: Centro de Estudios Interdisciplinarios, Jurídicos, Sociales y Humanistas (CIES), Facultad de Derecho y Ciencias sociales, Universidad Icesi.
\end{abstract}

\begin{abstract}
Resumen
A partir de la creación y divulgación de la ley 1448 del año 2011, se han desarrollado iniciativas encaminadas a garantizar el derecho a la verdad, justicia y reparación de las víctimas del conflicto armado. Sin embargo, no se ha desarrollado una sección destinada a la población LGBTI (o de orientaciones sexuales no normativas), que muestre las formas de reparación a la que tienen derecho. Dentro de esta población, las mujeres trans (transexuales, travestis y transformistas) son una población fuertemente afectada por el conflicto por su apariencia, tipos de trabajo y rechazo social. Por esto, el artículo busca trabajar el tema de reparación en mujeres trans víctimas del conflicto armado. Debido a su situación particular se considera importante trabajar inicialmente en la reparación simbólica, pues es el mejor camino para disminuir los niveles de exclusión antes, durante y después de la victimización, y reconstruir su proyecto de vida. Por
\end{abstract}

* Estudiante de Ciencia Política de la Universidad de los Andes (Bogotá, Colombia). 
lo anterior, la pregunta a resolver es ¿por qué no se han creado medidas de reparación simbólica para las mujeres trans víctimas del conflicto armado colombiano? Para responderla, se hará un análisis documental y entrevistas a mujeres trans víctimas y a tomadores de decisiones de políticas públicas y, aunque la respuesta no es final, se mostrará que las peticiones de estas mujeres se relacionan con una reparación que responda a la situación antes y después de su victimización.

\section{Palabras clave:}

Reparación simbólica, mujeres trans, víctimas, conflicto armado, género.

\section{Introducción}

Con la expedición de la ley 1448 (Ley de Víctimas y Restitución de Tierras) en el año 2011, se reconocieron a las víctimas del conflicto armado en Colombia y se decidió que éstas tenían derecho a la verdad, la justicia y la reparación por lo sucedido. Lo anterior, con el objetivo de lograr la reconciliación entre toda la sociedad colombiana y permitir la recuperación de la confianza y la construcción de un futuro compartido (Ley de víctimas y restitución de tierras, 2011). Por esto, se han desarrollado varias iniciativas dirigidas a garantizar estos derechos; sin embargo, al ser un proceso que lleva poco tiempo, aún hay varios aspectos en los que se debe mejorar. Este es el caso de la reparación simbólica, tema que presenta ciertos vacíos y nuevos retos. Por lo anterior, el tema de esta investigación es la reparación simbólica desde una perspectiva de género. Se centrará en las mujeres trans (transexuales, travestis y transformistas) víctimas del conflicto armado.

La reparación simbólica es un elemento importante en la reparación integral. Lo anterior, porque durante el conflicto armado la victimización cobra formas tanto físicas como psicológicas. Así, al pensar en mejorar las condiciones de las víctimas el aspecto simbólico es una forma de contribuir con la disminución del sufrimiento y la reconstrucción de los proyectos de vida. Ahora bien, a la hora de reparar también se deben tener en cuenta las formas de victimización y las diferentes maneras en que se vive una misma situación. Es por esto que diferentes grupos han solicitado la reparación diferenciada de mujeres, niños, indígenas y afros al ser victimizadas y recordar esta victimización de forma diferente (Grupo Memoria Histórica, 2011). Bajo este reclamo, se han iniciado distintas medidas que permiten entender la situación particular de diferentes comunidades y que tienen un efecto reparador simbólico. Este es el caso de obras de teatro, creación de canciones, conciertos, presentaciones en museos que cuentan lo que sucedió 
en diferentes regiones del país, actos de regreso a sus tierras o entierro de seres queridos mediante rituales (Centro Nacional de Memoria Histórica), creación de monumentos, disculpas públicas de parte del Estado y de algunos grupos armados, atención psicosocial y actividades que responden a las vivencias particulares de cierta comunidad de víctimas. Sin embargo, en todas estas medidas no se ha conocido aún una dirigida a un grupo que también ha sido victimizado de forma particular durante la guerra y que, como todos los grupos sociales, tiene el derecho a ser reparada: las mujeres trans.

Por lo anterior, la pregunta que se busca responder es: ¿por qué no se han iniciado medidas de reparación simbólica para las mujeres trans víctimas del conflicto armado colombiano? Para responder esta pregunta se usó una metodología cualitativa que consistió en la revisión de documentos y en la realización de entrevistas a mujeres trans, miembros de la unidad de víctimas y organizaciones que trabajan con estas mujeres. A causa del poco tiempo que lleva esta investigación, las entrevistas se hicieron solamente en Bogotá. Sin embargo, con el tiempo se espera llegar a otras ciudades (como Barranquilla, Cali, Medellín) o pueblos, que permitan ver la problemática desde una perspectiva más local. Para desarrollar lo anterior, el texto estará dividido en cuatro secciones. En la primera se hará una breve revisión de literatura que busca definir los conceptos clave; en la segunda se mostrará lo que se ha hecho y lo que no desde el Estado por lograr esta reparación; en la tercera se mostrará lo que han hecho las organizaciones no gubernamentales y finalmente se mostrará el punto de vista de las mujeres trans víctimas, lo que permitirá llegar a una conclusión.

Antes de desarrollar cualquier argumento, es importante hacer una explicación de lo que es ser trans. Trans es una identidad de género diferente a la de hombre o mujer, razón por la cual tiene caracterísiticas específicas, que provocan la construcción de una categoría distinta a las ya existentes. Así, una persona trans es aquella que nace de un sexo pero que se identifica con el género opuesto. Pueden haber hombres trans (personas que nacen con sexo femenino pero que se identifican con el género masculino), mujeres trans (personas que nacen con sexo masculino pero que se identifican con el género femenino) $\mathrm{u}$ otras personas que aunque no se identifican con el género asignado, no se consideran hombres o mujeres (Colombia Diversa, 2014). Esta investigación se centró en las mujeres trans por la particularidad de su victimización y por el registro que se tiene. Esto no 
quiere decir que no hayan hombres trans víctimas y que su reparación no sea igual de importante. Ahora bien, al hablar de identidad de género es importante dar una definición. Gabriela Castellanos (2006) lo define como la realidad psíquica de cada individuo, que es construida sin que coincida necesariamente con sus características físicas y su sexo. A esto, Colombia Diversa (2014) agrega que esta construcción puede estar acompañada por la vivencia personal del cuerpo (el cual puede ser modificado según elija cada persona) y por otras expresiones de género como la vestimenta, el modo de hablar y los modales.

Ahora bien, para afirmar que es necesaria una reparación simbólica de este grupo social, se debe primero mostrar la forma en que fue victimizado. La más conocida es el desplazamiento, que se da por tres razones principales: amenazas contra su identidad de género, riesgo de reclutamiento forzado y dificultades del entorno socio-político (Prada, Herrera, Lozano y Ortiz, 2012). Sin embargo, hay otras formas de victimización que afectan igual o más la integridad de la víctima. En primer lugar, se cuenta la violencia sexual y fuertes golpizas que se hacen bajo el argumento de que están ayudando a estas mujeres a corregir su orientación sexual (Mujeres al Borde, 2013). En segundo lugar, la estigmatización realizada por los grupos armados, que reproduce la discriminación dentro de las comunidades en las que viven estas mujeres y dificultan el desarrollo de su vida (Colombia Diversa, 2009). En tercer lugar la violencia por intolerancia (mal llamada limpieza social), donde matan a diferentes grupos sociales y uno de los principales objetivos es la mujer trans (Rojas 1996; Fernández y Otero 2009; Albarracín y Rincón, 2013). La justificación principal es recordar el estado de inferioridad o subordinación, o para liquidar lo que el otro cuerpo representa (Colombia Diversa, 2014).

En cuarto lugar se han encontrado casos de reclutamiento de estas mujeres de parte de grupos armados, donde las mujeres trans son obligadas a cumplir el rol de hombres en la tareas a desarrollar dentro de las filas, pero violentadas sexualmente por reconocerse a sí mismas como mujeres (Prada et al., 2012). Asimismo, se pueden encontrar casos sistemáticos de violencia en una región, como es el caso de las peleas de boxeo para homosexuales y mujeres trans, organizadas por Marco Tulio Pérez Guzmán (alias el Oso) en San Onofre, como forma de burla y rechazo social (información suministrada por Nancy Prada, investigadora del Centro Nacional de Memoria Histórica). Finalmente, es importante nombrar la existencia de homicidios selectivos de esta población, al ser la 
modalidad de violencia que más muertos ha provocado durante el conflicto armado pero la que menos visibilidad tiene, a causa de su carácter individual (Albarracín et al., 2013).

Con todas estas formas de victimización se evidencia que éstas no son solamente físicas ni provienen únicamente de los grupos armados, sino que también se hace un ataque simbólico (al crear estigmatización) y se desarrollan prácticas violentas desde la comunidad. Tomando a Albarracín et.al (2013), los ataques por medio del lenguaje y los crímenes de "eliminación física" crean un ambiente de invisibilización de esta población. $\mathrm{Y}$ esta invisibilidad es una forma de violencia simbólica, al ser una violencia insensible que se ejerce por medio de caminos de comunicación y del desconocimiento u ocultamiento de los crímenes realizados (Albarracín et al., 2013). Por esta razón, se vuelve imperativo pensar en una reparación simbólica dirigida a las víctimas, al ser una forma de visibilización de lo ocurrido y de reconocer del sufrimiento vivido por esta población.

Teniendo claro el tema de mujeres trans, se hará una explicación de lo que se entiende por reparación simbólica. Ésta hace parte de un conjunto de medidas que se buscan implementar en un marco de justicia transicional; lo cual se refiere e arreglos judiciales y extrajudiciales que permiten la transición de una situación de guerra a una de paz (Rettberg, 2005), y en el cual se debe atender los derechos de las víctimas a la verdad, justicia, reparación y garantías de no repetición (Ciurlizza, 2012). De estos derechos, la reparación se define habitualmente como el acto de devolver a las víctimas a la situación previa a la victimización (Sassòli, 2009). Sin embargo, Uprinmy y Saffon (2009) han desarrollado otro concepto, conocido como "reparación transformadora", en donde se argumenta que las medidas de reparación no deben devolver a la situación previa a las víctimas, pues ésta es precaria, sino que debe ser una oportunidad para superar la exclusión y la desigualdad y lograr una transformación democrática de las sociedades.

Ahora bien, la reparación puede darse de diferentes formas. Dentro de las principales se encuentran la restitución, compensación, satisfacción y garantías de no repetición. En esta ocasión se explicará la forma de satisfacción, que es equivalente a la reparación simbólica. Ésta se define en la ley 1448 de 2011 como "toda prestación realizada a favor de las víctimas o de la comunidad en general que tienda a asegurar la preservación de la memoria histórica, la no repetición de los hechos victimizantes, la aceptación pública de los hechos, la solicitud de perdón público y el restablecimiento de la dignidad de 
las víctimas". Diferentes autores resaltan la importancia de reparar a las víctimas simbólicamente y no sólo económicamente, pues consideran que el punto de partida en la reparación es el reconocimiento de la dignidad de las víctimas, al ser esta una forma de legitimar sus historias y vivencias (Orjuela, 2009; Rebolledo y Rondón, 2010; Tejada, 2011). De igual forma, explican que es necesario reconocer el dolor causado y la forma como es vivido e interpretado por cada víctima, pues es de esta forma que se reinventa la verdad, se construyen las identidades y se define la forma en que la víctima quiere ser reparada. Además, al reconocer su dolor y buscar el reestablecimiento de su dignidad, las víctimas pueden recuperar su condición de ciudadanos y ciudadanas con derechos y con la capacidad de participar en la vida pública, lo que les permite reconstruir su proyecto de vida (Orjuela, 2009; Rebolledo y Rondón, 2010; Tejada, 2011).

Teniendo lo anterior, a continuación se busca entonces entender por qué no se han iniciado medidas de reparación simbólica para las mujeres trans víctimas del conflicto armado, teniendo en cuenta que han vivido victimizaciones que ameritan una atención diferenciada. Al buscar responder esto, se pretende también problematizar la visión que ha prevalecido dentro de las leyes estatales. Para iniciar, se hablará de la Ley 1448 de 2011, al ser este el punto de partida para la reparación de las víctimas en Colombia. En esta ley se introducen tres normas que hacen visibles a las víctimas LGBTI del conflicto armado: reconocimiento de las parejas del mismo sexo como víctimas; mención de la orientación sexual dentro del principio de igualdad; y establecimiento de la orientación sexual como parte del enfoque diferencial (Albarracín et al, 2013). Sin embargo estos elementos, aunque importantes, no reconocen la existencia de una identidad de género diferente a la de hombre y mujer, lo que deja de lado nuevas formas de entender la victimización y la forma en que el género trans la vive. Al hablar de enfoque de género en la Ley de Víctimas sólo se contempla la atención diferenciada que deben tener las mujeres y se deja de lado el género trans. En las entrevistas realizadas a mujeres trans víctimas de desplazamiento, se conocieron vivencias que atacan la identidad de género de estas mujeres y, por tanto, es importante contemplar un reparación que atienda a sus necesidades. Katy, desplazada desde el año 2007 de Algesiras (Huila) por la guerrilla, muestra cómo lo vivido en su pueblo le produjo traumas psicológicos.

Yo estoy traumatizada psicológicamente con esas personas. Vivir allá en ese pueblo 
para mi fue algo terrible porque allá se entraba la guerrilla cada ratico, allá te amanazaban por todo (...). Una vez a mi me mandaron a cortar el cabello, me dijeron que si yo tenía vagina, que yo qué era lo que tenía, que yo quién era. Me iban a mandar a calvear, no eso fue terrible (Katy, 23 de agosto de 2014).

Esta invisibilización del género trans en la Ley de Víctimas trae como consecuencia otros problemas que reproducen esta invisibilidad. Este es el caso de la ruta de reparación planteada por la Unidad de Atención y Reparación Integral a las Víctimas (UARIV). Para que una persona sea reconocida como víctima y tenga el posterior derecho a la reparación, debe haber llenado el Formato Único de Declaración (FUD), creado en 2011. Y antes sólo existía el Sistema de Información para la Población Desplazada (SIPOD). En estos formatos, antes del 2012, sólo se preguntaba por el sexo y las dos opciones eran femenino-masculino (Suayan Barón, 4 de septiembre de 2014). Este fue el caso de Katy, pues al llegar a Bogotá en el año 2007 se registró en el SIPOD, donde sólo pudo marcar sexo masculino. Con esto, no hubo un reconocimiento de su condición y por tanto hay una dificultad de parte del Estado para ubicar a esta persona y posteriormente garantizarle su derecho a la reparación (caso que se replica en muchas otras personas).

Después del año 2012, en el FUD se cambió la variable sexo y se agregaron tres opciones: hombre-mujer-LGBTI. Esto supone un registro donde se toma a la comunidad LGBTI como una homogénea y que vive la victimización de igual forma, dejando de lado el tema identitario, que es un tema de vital importancia para las mujeres trans, al ser la forma en la que ellas se reconocen y esperan ser reconocidas por la sociedad. Hasta este año se ha contemplado desagregar esta opción para tener un registro detallado del universo de víctimas y se espera que sea implementado en poco tiempo (Suayan Barón, 4 de septiembre de 2014). Como se puede ver, el aspecto técnico del registro de víctimas es un problema que ha causado la "eliminación" de las mujeres trans en el universo de víctimas colombianas, algo que seguramente ha dificultado que estas personas sean sujetos de reparación, pues el que no diligencia el FUD no aparece dentro del universi de víctimas con derecho a atención integral. Este es el caso de Claudia (mujer trans desplazada de ciudad bolivar en enero de este año) y de Balery (mujer trans desplazada de Sucre en febrero de este año, por la banda los rastrojos). En el primer caso, Claudia no fue registrada como víctima por tratarse de un desplazamiento intraurbano, algo que desconoce 
su situación y la forma en que fue sacada de su lugar de residencia. En el segundo caso, Balery se registró en el FUD pero no aparece en el Registro Único de Víctimas (RUV). En los dos casos, sus problemáticas no les permite entrar a un posible proceso de reparación ni ser reconocidas por el Estado como víctima del conflicto armado.

\begin{abstract}
Cuando yo llegué a Bogotá, me registré como víctima de desplazamiento pero no sé por qué no aparecí registrada. Yo tengo todos mis papeles y un abogado me está ayudando. Le pusimos una tutela a la UARIV, pero no he tenido lo del transporte para ir a hablar con el abogado y ver cómo va eso (Balery, 6 de septiembre de 2014).
\end{abstract}

Siguiendo con la ruta de reparación, después del registro que se hace en el FUD, se implementa el Plan de Atención, Asistencia y Reparación Integral a Víctimas del Conflicto Armado (PAARI). Con esto se pueden identificar las condiciones socioeconómicas, psicosociales, familiares y nutricionales de las personas víctimas y se pueden remitir a la oferta institucional que se encuentra dentro del SNARIV. El PAARI sí contiene las variables de sexo, orientación sexual e identidad de género, lo que permite que las personas que no pudieron identificarse como mujeres trans en el FUD, sí lo puedan hacer en este segundo instrumento en donde aparecen 553 mujeres trans en todo el país (Suayan Barón, 4 de septiembre de 2014). En esta investigación no se encontró una persona que estuviera registrada en el PAARI; sin embargo, para el caso de Katy, quién fue desplazada hace siete años, surge la pregunta de por qué no la han contactado para conocer su situación e iniciar un proceso de reparación, dentro del que se encuentra la simbólica (según Suayán Barón la Unidad se acerca a ellos a partir del registro que se tiene en el RUV).

Ahora bien, es importante nombrar la Resolución 0388 del 2013 (Protocolo de Participación Efectiva de las Víctimas del Conflicto Armado), en donde se crea el marco para garantizar la participación efectiva de las víctimas en la planeación, ejecución y control de las políticas públicas; así como garantizar a las víctimas su intervención real y efectiva en los espacios de participación ciudadana. Para esto, ordenan la creación de mesas de participación a nivel municipal, departamental y nacional, en donde se incluye la presencia de representantes de la comunidad LGBTI. En la creación de esta resolu- 
ción hubo participación de la Corporación Opción, en cabeza de Diana Navarro, mujer trans desplazada de Medellín que trabaja por el reconocimiento de los derechos de estas personas. Este protocolo es un paso muy importante a la hora de pensar en la inclusión de esta comunidad; sin embargo, dificulta la representación de todos sus miembros pues, como se dijo anteriormente, esta comunidad no es homogenea ni vive la victimización de igual forma. Asimismo, Diana afirma que durante su intervención en la creación de este Protocolo hubo ciertos desacuerdos.

Una discusión que yo tuve siempre al interior de la representación es que hacía falta implementar un enfoque diferencial. Ellos no entendian qué era enfoque diferencial. (...) El desplazamiento del que yo fui víctima no es el mismo que el de otra persona; son diferentes causas y diferentes problemáticas que me causan a mi (...). Enfoque diferencial unicamente lo entendían como una cuota de participación, no un eje transversal para los temas de reparación, en cómo van a reparar el daño que se hizo a una persona que es víctima del conflicto armado (Diana Navarro, 2 de septiembre de 2014).

Según lo que cuenta Diana, esta concepción de enfoque diferencial puede dificultar la manera en que se concibe la atención a las víctimas con identidades de género distintas. Para el caso de Bogotá, al hablar con el representante de la mesa distrital, Julian Pantoja, se observó que no hay una representación efectiva de las mujeres trans en esta mesa, pues la comunicación entre él y esta población no ha sido activa. Esto produce que las ideas expresadas por el representante puedan no ser lo que las víctimas de esta comunidad desean y por tanto no hay una garantía completa de reparación para ellas.

Uno de mis grandes proyectos es hacer un gran encuentro distrital con la población LGBT, primero porque yo represento a una población a la cual no conozco (...). Lo que yo le pido a la Unidad de Víctimas es que comboque a las personas, pero ellos se basan en los principios de confidencialidad para ponerme trabas. Yo no voy a divulgar su información confidencial, lo único que quiero es conocer a quien represento, que ellos conozcan a su representante e iniciar un diálogo donde se intercambien experiencias y yo pueda saber lo que han hecho y necesitan estas personas (Julian Pantoja, 20 de septiembre de 2014). 
Finalmente, es importante hacer un pequeño apéndice con la labor que está realizando en Centro Nacional de Memoria Histórica, quien es la entidad encargada de desarrollar una reparación simbólica en términos de búsqueda de la verdad, creación de memoria histórica y de implementación de actividades pedagógicas y museísticas que divulguen lo ocurrido durante el conflicto armado (Centro Nacional de Memoria Histórica, 2014). Hasta el momento, se ha reconocido a la comunidad LGBTI como víctimas de especial atención, y se dedicó un aparte del informe Basta Ya! para explicar su situación. Adicional a eso, actualmente se está desarrollando una investigación que busca esclarecer lo ocurrido en La Playa de Alto de Julio (San Onofre, Sucre), donde alias el Oso organizó peleas de boxeo entre personas homosexuales y mujeres trans. Sin embargo, hasta el momento no se tiene registro de alguna iniciativa simbólica dirigida a las mujeres trans.

Luego de discutir las dificultades a nivel estatal, se hará un recuento sobre la labor de las organizaciones que trabajan con mujeres trans víctimas. En general, estas organizaciones no trabajan sólo con esta población, sino que incluyen a estas personas dentro de su trabajo. Este es el caso de Caribe Afirmativo (de Barranquilla), Fundación Santa María (de Cali), Procrear, grupo GAT, Corporación Opción y Parces (de Bogotá). Para el caso de Bogotá, las funciones principales de estas organizaciones han sido la denuncia de los abusos contra las mujeres trans, de la vulneración de sus derechos y la visibilización y empoderamiento de éstas en diferentes espacios. Adicionalmente, brindan ayuda a personas que llegan de las regiones a construir su apariencia física de acuerdo a la forma como se identifican. Así, contribuyen a iniciar o finalizar procesos que les permita cumplir con sus deseos por transitar del género masculino al femenino (Laura Weins, 28 de agosto de 2014). Finalmente, en ciertos casos brindan ayuda psicosocial a mujeres trans que lo necesiten y tienen trabajo en cárceles, donde también se encuentran víctimas del conflicto armado (Diana Navarro, 2 de septiembre de 2014).

Con todo lo anterior, se puede observar que no se han desarrollado iniciativas que inicien un proceso de reparación simbólica para las mujeres trans víctimas del conflicto armado, debido a diferentes trabas o dificultades presentes en el ámbito estatal. Sin embargo, es importante indagar en el punto de vista de las víctimas y observar sus iniciativas. En primer lugar, es importante nombrar una reunión organizada por el grupo de apoyo trans GAT, apoyado por Redepaz, Paiis y la Universidad de los 
Andes, en donde se hizo un evento llamado "NuesTrans voces en la Habana" cuyo objetivo fue reunir ideas sobre cómo las mujeres trans deben ser incluidas en los diálogos de la Habana y cuáles son sus propuestas para lograr la paz. Dentro de éstas, se encuentra el reconocimiento del dolor de estas personas durante la guerra, no sólo por los ataques recibidos sino por la desaparición o asesinato de sus familiares a manos de grupos armados. Asimismo, se han desarrollado otras iniciativas en Bogotá, como la semana por la paz, seminarios de no violencia y el homenaje a Wanda Fox (mujer trans asesinada en el barrio Santafé), que no son desarrolladas desde el Estado pero que sí contribuyen a reparar simbólicamente a esta población.

Por otro lado, al hablar con varias mujeres trans y escuchar a las que participaron del evento anteriormente descrito, se encontró que la petición más grande que tienen es el de la oportunidad de recibir educación en todos los niveles, al ser esta una posibilidad de cambiar de actividades a las que son normalmente marginadas (prostitución y peluquería).

Pues la verdad una no quiere que la mantengan, pero por ejemplo en mi caso, yo siempre he soñado estudiar en una universidad, ser profesional. (...) Yo aprendi a la fuerza a ser estilista, cosa que agradezco, pero allá recibí puras humillaciones, hasta que dije ay no yo no quiero sufrir más y entonces me metí en la prostitución (Katy, 23 de agosto de 2014).

Teniendo en cuenta que la definición de reparación simbólica en la Ley de Víctimas y Restitución de Tierras se contempla como estrategias que preserven la memoria histórica, la no repetición de los hechos victimizantes, la aceptación pública de los hechos, la solicitud de perdón público y el restablecimiento de la dignidad de las víctimas, en las conversaciones que se desarrollaron, muchas de estas mujeres esperan otras cosas además de las nombradas allí. Para este caso particular, la reparación no se puede garantizar sólo con asegurar que no vuelvan a ser atacados por grupos armados, que éstos o el Estado pidan perdón y que se sepa lo que les ocurrió, pues como se ha dicho anteriormente, la discriminación también se da desde la sociedad.

Si me pregunta qué es lo que yo necesito pues yo le digo que necesito ayuda psicológica, lo que me pasó ha sido muy difícil de entender y sobrellevar. Quiero un empleo, llevo desde febrero sin poder trabajar y yo sé hacer cosas, yo estoy 
jóven. Yo soy chef, pero nadie me da trabajo y yo nunca aprendí a ser peluquera y como prostituta no podría trabajar. Quiero volver a mi costa, extraño el calor. Pero no quiero que me pidan disculpas los que me hicieron esto, a ellos no los quiero ver nunca más (Balery, 6 de septiembre de 2014).

A manera de conclusión, se ve que para que las mujeres trans puedan recibir una reparación que responda a sus vivencias particulares, se deben iniciar medidas estatales en términos legales y prácticos, que permitan la inclusión de esta población y su reconocimiento como víctima. Reconocer su identidad de género y brindarle espacios de participación son puntos centrales, al ser dos problemáticas que se visibilizaron durante la investigación. Adicionalmente, al hablar con esta población se encontró que más que pedir disculpas, hacer monumentos o museos (que no dejan de ser importantes), ellas le dan prioridad a otras medidas que aseguren el mejoramiento de su calidad de vida, donde puedan participar en más espacios, salir de los lugares comunes a las que son llevadas por causa de la discriminación (sectores de prostitución y peluquerías), la eliminación de estereotipos y el reconocimiento de sus derechos como ciudadanas colombianas.

En este sentido, y tomando la teoría de Uprinmy y Saffon (2009) se debería pensar en una reparación simbólica transformadora, pues no se puede pensar en devolver a las víctimas a la situación en la que estaban, al ser precaria, invisibilizada y poco atendida, sino que debe pensarse en mejorar esta situación. Esta reparación se puede contemplar como una serie de actos o iniciativas que faciliten la integración de estas mujeres a la vida social, en condiciones de igualdad, para darles así la posibilidad de participar activamente en la sociedad. Igualmente, se podría pensar en la reconstrucción de las identidades de género de estas mujeres, que en ocasiones pueden verse afectadas por la victimización, para que de esta manera ellas puedan cumplir el proyecto de vida que desean.

Aunque lo anterior no es una conclusión contundente, sí abre una puerta para pensar que las mujeres trans necesitan elementos distintos a lo que se ha pensado a la hora de hablar de reparar a las víctimas del conflicto armado. Se espera que esta investigación siga avanzando y se puedan encontrar más respuestas que contribuyan al inicio y desarrollo de una reparación simbólica para estas mujeres. 


\section{Bibliografía}

Acción social (2011). Ley de Víctimas y Restitución de Tierras (Ley 1448 de 2011).

ALBARRACÍN, Mauricio; Rincón, Juan. (2013). De las víctimas invisibles a las víctimas dignificadas: los retos del enfoque diferencial para la población LGBTI en la Ley de víctimas. Revista de Derecho público, 31: 5-31. ISSN: 1909-7778.

CASTELLANOS, Gabriela. (2006). Sexo, género y feminismo: tres categorías en pugna. Cali: Centro de estudios de género, mujer y sociedad.

CIURLIZZA, Javier. (2012). Justicia transicional en Colombia: un modelo para desarmar. En A. Rettberg (Comp.). Construcción de paz en Colombia (pp. 89-116). Bogotá, Colombia: Ediciones Uniandes.

CENTRO NACIONAL DE MEMORIA HISTÓRICA. (2011). Mujeres que hacen historia: las luchas por la reinterpretación de la violencia y la discriminación de género. La memoria histórica desde la perspectiva de género: conceptos y herramientas (pp. 2544). Bogotá, Colombia: $\mathrm{CMH}$

CENTRO NACIONAL DE MEMORIA HISTÓRICA. (2014). ¿Qué es el Centro Nacional de Memoria Histórica? Consultado el 1 de septiembre de 2014, desde http://www. centrodememoriahistorica.gov.co/somos-cnmh/que-es-el-centro-nacional-de-memoriahistorica.

COLOMBIA DIVERSA. (2014). Cuando el prejuicio mata: Informe de Derechos Humanos de personas LGBT en Colombia. Consultado de 29 de agosto de 2014, desde http://www.colombia-diversa.org/2014/06/cuando-el-prejuicio-mata-informe-de.html.

COLOMBIA DIVERSA. (2009). Algunas intuiciones sobre las violaciones a los derechos humanos de la población LGBT en el marco del conflicto armado. Derechos humanos de lesbianas, gays, bisexuales y transgeneristas en Colombia 2006-2007 (pp. 345-356). Bogotá, Colombia: Colombia Diversa.

FERNÁNDEZ, Carlos H. y Otero, Silvia. (2009) Panfletos y limpieza social. Efectos mortales y no mortales. Revista Cien días vistos por CINEP. 67. 
MUJERES AL BORDE. 2013. Informe sobre Colombia: 16 ronda del examen periódico universal. Consultado el 18 de julio de 2014, desde http://www.upr-info.org/IMG/ pdf/js7_upr_col_s16_2013_jointsubmission7_s.pdf.

ORJUELA, Fernando. (2009). Reparar con símbolos. Bogotá: Universidad Nacional de Colombia (Documento en pdf).

PRADA, Nancy; Herrera, Susan; Lozano, Lina y Ortiz y Ana M. (2012). Mejor me voy antes de que me maten. ¡A mí me sacaron volada de allá! Relatos de vida de mujeres trans desplazadas forzosamente hacia Bogotá (pp. 111-122). Bogotá, Colombia: Universidad Nacional de Colombia.

REBOLLEDO, Olga; Rondón, Lina. (2010). Intervención psicosocial con fines de reparación con víctimas y sus familias afectadas por el conflicto armado interno en Colombia: equipos psicosociales en contextos jurídicos. Revista de estudios sociales, 36 40-50. ISNN 1900-5180.

RETTBERG, Angelika. (2005). Reflexiones introductorias sobre la relación entre construcción de paz y justicia transicional. En A. Rettberg (comp.) Entre el perdón y el paredón: preguntas y dilemas de la justicia transicional (pp. 1-15).

ROJAS, Carlos E. (1996). Contra quiénes se dirige. La violencia llamada 'Limpieza social"’ (pp. 25-45). Bogotá, Colombia: Cinep.

SASSÒLI, Marco. (2009). Reparation. En V. Chetail (edit.). Post-conflict Peacebuilding: A lexicon (pp. 369-379). Oxford, Inglaterra: Oxford University Press.

TEJADA, Carolina. (2011). Dignidad humana y rehabilitación. Lecciones en torno a la atención integral de las víctimas de la violencia. Seminario Internacional. Desafíos para la reparación integral a las víctimas del conflicto armado interno en Colombia (pp. 139-156).. Bogotá, Colombia: Alcaldía Mayor de Bogotá.

UNIDAD PARA LA ATENCIÓN Y REPARACIÓN INTEGRAL A LAS VÍCTIMAS (10 de mayo de 2013). Resolución 0388 de 2013 por la cual se adopta el Protocolo de Participación Efectiva de las Víctimas del Conflicto Armado. Consultado el 29 de agosto de 2014, desde http:/www.unidadvictimas.gov.co/images/docs/RESOLU- 
CION_0388_DE_10_DE_MAYO_DE_2013.pdf.

UPRIMNY, Rodrigo; Saffon, María P. (2009). Reparaciones transformadoras, justicia distributiva y profundización democrática. Reparar en Colombia: los dilemas en contextos de conflicto, pobreza y exclusión (pp. 31- 70). Bogotá: ICTJ y DeJuSticia

Entrevistas

Balery. Comunicación personal (6 de septiembre de 2014).

Claudia. Comunicación personal (30 de agosto de 2014).

Diana Navarro. Comunicación personal. (2 de septiembre de 2014).

Julian Pantoja. Comunicación personal. (20 de septiembre de 2014).

Katy. Comunicación personal (23 de agosto de 2014).

Laura Weins. Comunicación personal (28 de agosto de 2014).

Suayan Barón. Comunicación personal (4 de septiembre de 2014). 
